\title{
Efeito de bordas lineares na fenologia de espécies arbóreas zoocóricas em um remanescente de Mata Atlântica'
}

\author{
Gabriela Reznik², Jakeline Prata de Assis Pires ${ }^{3}$ e Leandro Freitas ${ }^{2,4}$
}

Recebido em 12/08/2011. Aceito em 28/11/2011

\begin{abstract}
RESUMO
(Efeito de bordas lineares na fenologia de espécies arbóreas zoocóricas em um remanescente de Mata Atlântica). A dispersão de diásporos é uma das fases cruciais do ciclo de vida vegetal e a dinâmica fenológica das espécies está entre os fatores reguladores do modo de dispersão e da atividade dos dispersores. Em paisagens sujeitas à fragmentação, a distribuição das síndromes de dispersão pode ser modificada devido às mudanças na composição e estrutura da flora, causadas pelo efeito de bordas, que também podem provocar alterações na fenodinâmica das plantas. Neste estudo, as síndromes de dispersão (zoocoria, anemocoria e autocoria) de 162 espécies arbóreas foram identificadas e, para 57 dessas espécies, esta informação foi relacionada a diferentes medidas de fenologia de frutificação (índice de atividade, percentual de intensidade de Fournier e intensidade ponderada por área basal) em ambientes de bordas lineares e interior de mata. Em todos os ambientes houve predominância de espécies zoocóricas e, em geral, a distribuição das síndromes não diferiu entre os ambientes. Considerando os três ambientes conjuntamente, espécies zoocóricas e anemocóricas frutificaram ao longo de todos os meses, enquanto as autocóricas apresentaram meses sem frutificação. Quando os ambientes foram analisados separadamente, a fenodinâmica de frutificação das espécies zoocóricas ( $\mathrm{N}$ = 44) diferiu entre os três ambientes. Os resultados indicam a existência de efeitos de borda na fenologia de frutificação das espécies dispersas por animais, apesar da ausência desses efeitos na distribuição das síndromes de dispersão. Isso sugere que a passagem de redes elétricas e gasodutos em remanescentes de Mata Atlântica pode afetar a dinâmica temporal de disponibilidade de frutos para frugívoros, com possíveis consequências para as interações planta-dispersor, mesmo quando não acarretam diminuição na oferta total de recursos para esses animais.
\end{abstract}

Palavras-chave: dispersão de sementes, florestas tropicais, fragmentação de habitats, produção de frutos

\begin{abstract}
(Effect of linear edges in the phenology of animal dispersed tree species in a remnant of Atlantic Forest). The dispersal of seeds is one of the crucial phases of the life cycle of plants and plant phenology is among the factors that regulate the dispersal mode and activity of dispersers. In landscapes subject to fragmentation, the distribution of dispersal syndromes may be modified due to changes in flora composition and structure caused by edge effects, which may also change plant phenodynamics. In this study, the dispersal syndromes (zoochory, anemochory and autocory) of 162 tree species were identified and, for 57 of these species, this information was related to different measures of fruiting phenology (activity index, percentage of Fournier intensity and intensity weighted by basal area) in environments of linear edges and forest interior. In all environments there was a predominance of animal dispersed species and, in general, the distribution of syndromes did not differ among environments. Considering the three environments together, zoochorous and anemochorous species had continuous fruiting, while the autochorous fruiting pattern was intermittent. When the environments were analyzed separately, the fruiting phenodynamics of animal dispersed species $(\mathrm{N}=44)$ differed among the three environments. The results indicate the existence of edge effects on fruiting phenology of species dispersed by animals, despite the absence of these effects in the distribution of dispersal syndromes. This suggests that the passage of electric transmission lines and gas pipelines in remnants of Atlantic Forest may affect the temporal dynamics of fruit availability for frugivores, with possible consequences for plant-seed disperser interactions, even when it does not lead to a decrease in the total supply of resources for these animals.
\end{abstract}

Key words: fruit production, habitat fragmentation, seed dispersal, tropical forests

\footnotetext{
Parte da monografia de Iniciação Científica do primeiro Autor

Jardim Botânico do Rio de Janeiro, Rio de Janeiro, RJ, Brasil

Pontifícia Universidade Católica do Rio de Janeiro, Curso de Ciências Biológicas, Rio de Janeiro, RJ, Brasil

4 Autor para correspondência: leandro@jbrj.gov.br
} 


\section{Introdução}

A dispersão de diásporos, saída dos frutos e sementes da planta-mãe, é uma das fases cruciais do ciclo de vida vegetal (van Schaik et al. 1993). Em florestas de clima tropical úmido e de baixa sazonalidade climática, animais são os vetores de dispersão mais importantes e grande parte dos frutos das espécies arbóreas é consumida e dispersa por frugívoros, especialmente mamíferos e pássaros (Almeida-Neto et al. 2008; Du et al. 2009). A atividade dos animais dispersores não se restringe à remoção das sementes, mas também influencia a distribuição espacial e a dinâmica populacional das plantas, com impactos na estruturação das comunidades (Jordano et al. 2006). Especificamente para a Mata Atlântica, plantas que produzem frutos carnosos podem representar 90\% das espécies da comunidade arbórea (Galetti \& Pizo 1996), sendo que na sequência desta maior proporção de zoocoria (sensu van der Pijl 1982) ocorrem, respectivamente, anemocoria e autocoria (Talora \& Morellato 2000).

Padrões fenológicos das plantas podem influenciar o modo de dispersão e a atividade de dispersores (Morellato \& Leitão-Filho 1992; Reys et al. 2005) e alguns estudos correlacionaram as síndromes de dispersão com o comportamento fenológico da frutificação (Morellato et al. 2000; Talora \& Morellato 2000; Spina et al. 2001; Yamamoto et al. 2007; Du et al. 2009). A época de frutificação de espécies anemocóricas tropicais, por exemplo, vem sendo associada a variações em fatores abióticos, dada sua tendência a ocorrer nas estações mais secas, quando há menor umidade, maior velocidade dos ventos e maior eficiência na maturação dos frutos (desidratação do pericarpo em frutos secos) (Jara-Guerrero et al. 2011).

O processo de fragmentação de hábitats provoca alterações rápidas e drásticas na composição e na abundância de espécies arbóreas em florestas tropicais úmidas (Laurance et al. 2006) e essas alterações potencialmente podem afetar a composição e a abundância de espécies zoocóricas na comunidade. Já os efeitos de borda podem afetar a fenodinâmica das plantas, causando alterações nos padrões de atividade e intensidade de frutificação na comunidade (Ramos \& Santos 2005). Por sua vez, aves dispersoras de grandes sementes e mamíferos de grande porte são especialmente vulneráveis à perda de hábitat, o que pode levar ao risco de extinção local (Corlett \& LaFrankie 1998; Restrepo et al. 1999; Silva \& Tabarelli 2000; Tabarelli \& Peres 2002). Essas diversas modificações na composição e dinâmica da flora e da fauna advindas da fragmentação podem influenciar as interações bióticas, afetando funções ecológicas como predação, parasitismo, herbivoria, polinização e dispersão de sementes (Aizen \& Feinsinger 1994; Cunningham 2000). Por exemplo, o isolamento temporal causado pela falta de sincronia de floração entre indivíduos de uma determinada espécie, em ambientes perturbados, tem sido proposto como um fator proeminente para a redução na produção de frutos (Murawski et al. 1990; Doligez \& Joly 1997), o que, consequentemente, poderia gerar efeitos em cascata, afetando as interações com os dispersores.

Modificações na paisagem com a criação de estradas e redes de transmissão elétrica podem formar corredores desmatados no interior das florestas, classificados como bordas lineares (Murcia 1995). A abertura desses corredores promove maior exposição aos efeitos de borda, pois cria o dobro de bordas em sua extensão (Goosem 1997) e suas características determinam o grau de alterações microclimáticas próximas às bordas (Pohlman et al. 2007). Além disso, os efeitos destas bordas lineares podem variar em decorrência de seu período de existência, influenciando no grau de impacto da área devastada e na distância entre os fragmentos, o que afeta a conectividade entre eles (Kapos et al. 1997). Embora muito comuns nas paisagens tropicais, o conhecimento sobre efeitos de bordas lineares é ainda muito escasso. Devido à relativa menor área alterada e à proximidade entre fragmentos, é esperado que os impactos de bordas lineares sejam distintos daqueles advindos de bordas formadas por matrizes mais extensas.

Alguns estudos avaliaram os efeitos de borda em florestas tropicais sobre a fenologia das plantas (revisão em Laurance et al. 2003) ou sobre a distribuição das síndromes de dispersão (e.g., Tabarelli et al. 1999; Slik et al. 2011), mas pouco se sabe sobre as respostas combinadas dessas variáveis, o que permitiria avaliar os efeitos de borda sobre a variação temporal na disponibilidade de recursos aos frugívoros. Neste estudo foram registradas as síndromes de dispersão e a fenologia de frutificação das espécies arbóreas em bordas lineares e no interior florestal de um remanescente de Mata Atlântica, com o objetivo de: i) verificar se há diferenças na distribuição das síndromes de dispersão entre os ambientes de borda e interior; ii) descrever o comportamento fenológico do conjunto de espécies de cada síndrome; iii. verificar se a distribuição temporal de frutos zoocóricos difere entre os ambientes de borda e interior; e iv) verificar as diferenças no comportamento de distintos tipos de curvas fenológicas, as quais representam a presença das fenofases nas espécies, a intensidade das fenofases nos indivíduos e a intensidade das fenofases em relação ao volume de copa na comunidade.

\section{Material e métodos}

\section{Área de estudo}

O estudo foi realizado na Reserva Biológica União $\left(22^{\circ}\right.$ $27^{\prime} 30^{\prime \prime} \mathrm{S} \mathrm{e} 42^{\circ} 02^{\prime} 14^{\prime \prime} \mathrm{W}$ ), localizada entre os municípios de Casimiro de Abreu, Rio das Ostras e Macaé, estado do Rio de Janeiro, e com área de 2930 ha. Sua vegetação pode ser classificada como floresta ombrófila densa de terras baixas (sensu Veloso et al. 1991). A topografia é caracterizada pelos mares de morros, com elevação máxima de 376 m e solo tipo Latossolo Vermelho-Amarelo distrófico (Santos et al. 2006). O clima é predominantemente tropical úmido, sendo carac- 
terizado pelo sistema de classificação de Köppen como $A w$ tropical. Dados da última normal climatológica (1961-1990) obtidos na estação de Macaé (localizada ca. $20 \mathrm{~km}$ da área de estudo e a $32 \mathrm{~m}$ a.n.m.) caracterizam a estação menos úmida com menores temperaturas entre maio e setembro e a estação mais úmida com maiores temperaturas entre outubro e abril. A precipitação média anual de 1168,6 mm e temperatura média de $24^{\circ} \mathrm{C}$.

A área é entrecortada por dois corredores desmatados para passagem de rede de transmissão elétrica de alta tensão e gasoduto subterrâneo. O corredor formado pela rede elétrica se alarga por aproximadamente $100 \mathrm{~m}$ e foi implantado na década de 1960, enquanto o corredor formado pelo gasoduto possui largura de $25 \mathrm{~m}$ com implantação na década de 1980. Eles diferem quanto à matriz vegetativa, sendo o primeiro formado por vegetação arbustiva-arbórea de até 4 $\mathrm{m}$ de altura e com abundância de Pteridium arachnoideum (Kaulf.) Maxon (Dennstaedtiaceae) e o segundo por vegetação herbácea com predominância de gramíneas, mantida por constante manejo (Rodrigues 2004).

\section{Dados fenológicos}

Os dados fenológicos foram obtidos em nove parcelas (20 x $50 \mathrm{~m}$ ), anteriormente estabelecidas por Rodrigues (2004) para estudo de estrutura e dinâmica da comunidade arbórea $(\mathrm{DAP}>10 \mathrm{~cm})$. Das nove parcelas, três se localizam em bordas de gasoduto (GA), três em bordas de rede elétrica (RE) e três em interiores florestais (IN), distantes ao menos 400 $\mathrm{m}$ de qualquer borda. Para análise dos dados fenológicos, $\mathrm{o}$ presente estudo utilizou a base de dados do Programa Mata Atlântica - JBRJ, que abriga os valores de parâmetros fitossociológicos obtidos por Rodrigues (2004), após atualizações devido a mudanças nas identificações das espécies.

A atividade (Bencke \& Morellato 2002) e intensidade de Fournier (1974) para a fenofase fruto maduro foram registrados mensalmente, nas nove parcelas, entre agosto de 2006 e agosto de 2008 por Pires (2010). Foram amostrados, no total, 480 indivíduos, referentes a 182 espécies arbóreas de 43 famílias.

\section{Determinação das síndromes}

As espécies foram classificadas em três síndromes de dispersão - zoocoria, anemocoria e autocoria (van der Pijl 1982) - através de consulta a literatura taxonômica e estudos específicos sobre síndromes ou sistemas de dispersão (e.g., Cria-Fapesp 2003; Spina et al. 2001; Oliveira-Filho et al. 2004; Yamamoto et al. 2007). Os dados da literatura foram confirmados e revisados a partir de observação da morfologia dos frutos nas exsicatas depositadas no herbário RB, quando disponíveis. Apenas síndromes de dispersão primária (sensu van der Pijl 1982) foram consideradas.

\section{Análise dos dados}

O número e a proporção de espécies e indivíduos por síndrome foi obtido para a comunidade arbórea, sendo o mesmo procedimento realizado para os ambientes (GA, RE e IN) separadamente. A diferença entre proporção de espécies e indivíduos por síndrome foi testada pelo teste do Qui-quadrado de partição, utilizando o programa BioEstat 5.0.

Foi calculado o número de espécies de cada síndrome que frutificaram na comunidade e nos ambientes. A fenodinâmica das diferentes síndromes foi caracterizada por fenogramas para os índices de atividade (Bencke \& Morellato 2002), intensidade de Fournier (1974) e intensidade ponderada pela área basal (A.G. Silva et al. não publ. apud Pires 2010). O índice de intensidade ponderada pela área basal é uma adaptação do índice de Fournier e permite medir com maior acurácia a disponibilidade de recursos na comunidade, uma vez que representa a intensidade de frutificação de cada espécie considerando uma estimativa da área de copa de seus indivíduos (Pires 2010). A comparação entre os ambientes foi feita apenas para espécies zoocóricas, pois o número de espécies anemocóricas e autocóricas presentes na comunidade foi baixo. A fenodinâmica das espécies zoocóricas foi caracterizada por fenogramas para cada ambiente e a fenodinâmica das síndromes entre os ambientes foi comparada pelo teste $t$ pareado não-paramétrico de Wilcoxon, utilizando o programa Statistica 6.0.

\section{Resultados}

Houve predominância de zoocoria na comunidade e em cada ambiente (ca. 70\%, Tab. 1). No geral, a frequência das síndromes de dispersão não diferiu entre os ambientes, tanto para espécies quanto para indivíduos, sendo que a única exceção foi a menor frequência de indivíduos anemocóricos em IN (Tab. 2, Partição GA+RE -IN: $\chi^{2}=8,8 p=0,003$ ). Vinte espécies $(11,0 \%)$ permaneceram indeterminadas quanto às síndromes. A maioria dessas espécies foi identificada somente ao nível de família e não frutificou durante o período de observação, impossibilitando a identificação de sua síndrome.

Na comunidade, 44 espécies zoocóricas $(33,3 \%$ das espécies da síndrome), sete anemocóricas $(41,2 \%)$ e seis autocóricas $(46,2 \%)$ frutificaram. As espécies zoocóricas frutificaram ao longo de todos os meses de estudo, com três épocas de alta atividade de frutificação (i.e., ao menos três meses em que o número de espécies em fruto foi maior que a metade das espécies que frutificou no mês de pico), a saber, de setembro a novembro de 2006, maio a outubro de 2007 e janeiro a agosto de 2008 (Fig. 1A). Alta intensidade de Fournier (considerando o mesmo critério usado para atividade, ou seja, mais que metade da intensidade no pico de frutificação) se deu em duas épocas (setembro de 2006 a janeiro de 2007, e março a agosto de 2008), além de um pico isolado em junho de 2007 (Fig. 1D). Essas épocas de alta intensidade estiveram inseridas nas épocas de maior atividade de frutificação, exceto pela alta intensidade em dezembro de 2006 e janeiro de 2007, mas o pico de intensidade foi um mês antes que o pico de atividade (Fig. 1A e D). 
Gabriela Reznik, Jakeline Prata de Assis Pires e Leandro Freitas

Tabela 1. Número e proporção de espécies e de indivíduos zoocóricos, anemocóricos e autocóricos nos ambientes de borda de gasoduto (GA), borda de rede elétrica (RE) e interior de mata (IN) e considerando todos os ambientes (comunidade) na Reserva Biológica União, sudeste do Brasil.

\begin{tabular}{|c|c|c|c|c|c|c|c|c|}
\hline \multirow{3}{*}{ Síndrome } & \multicolumn{2}{|c|}{ Comunidade } & \multicolumn{6}{|c|}{ Ambiente } \\
\hline & \multirow{2}{*}{ Espécie } & \multirow{2}{*}{ Indivíduo } & \multicolumn{3}{|c|}{ Espécie } & \multicolumn{3}{|c|}{ Indivíduo } \\
\hline & & & GA & $\mathrm{RE}$ & IN & GA & $\mathrm{RE}$ & IN \\
\hline Zoocoria & $\begin{array}{c}132 \\
(72,5 \%)\end{array}$ & $\begin{array}{c}344 \\
(71,9 \%)\end{array}$ & $\begin{array}{c}70 \\
(72,9 \%)\end{array}$ & $\begin{array}{c}65 \\
(72,2 \%)\end{array}$ & $\begin{array}{c}63 \\
(77,8 \%)\end{array}$ & $\begin{array}{c}108 \\
(69,2 \%)\end{array}$ & $\begin{array}{c}123 \\
(72,4 \%)\end{array}$ & $\begin{array}{c}114 \\
(74,0 \%)\end{array}$ \\
\hline Anemocoria & $\begin{array}{c}17 \\
(9,3 \%)\end{array}$ & $\begin{array}{c}38 \\
(7,9 \%)\end{array}$ & $\begin{array}{c}10 \\
(10,4 \%)\end{array}$ & $\begin{array}{c}11 \\
(12,2 \%)\end{array}$ & $\begin{array}{c}3 \\
(3,7 \%)\end{array}$ & $\begin{array}{c}18 \\
(11,5 \%)\end{array}$ & $\begin{array}{c}16 \\
(9,4 \%)\end{array}$ & $\begin{array}{c}4 \\
(2,6 \%)\end{array}$ \\
\hline Autocoria & $\begin{array}{c}13 \\
(7,1 \%)\end{array}$ & $\begin{array}{c}74 \\
(15,4 \%)\end{array}$ & $\begin{array}{c}8 \\
(8,3 \%)\end{array}$ & $\begin{array}{c}8 \\
(8,9 \%)\end{array}$ & $\begin{array}{c}9 \\
(11,1 \%)\end{array}$ & $\begin{array}{c}22 \\
(14,1 \%)\end{array}$ & $\begin{array}{c}24 \\
(14,1 \%)\end{array}$ & $\begin{array}{c}28 \\
(18,2 \%)\end{array}$ \\
\hline
\end{tabular}

Tabela 2. Valores do teste de Qui Quadrado de partição $\left(\chi^{2}\right.$, g.l. $\left.=2\right)$ para a distribuição das síndromes de dispersão, por espécies e por indivíduos, entre os ambientes de borda de gasoduto, borda de rede elétrica e interior de mata na Reserva Biológica União, sudeste do Brasil. Valor em negrito representa diferenças significativas para $\mathrm{p}<0,05$.

\begin{tabular}{|c|c|c|}
\hline Espécies & $\chi^{2}$ & $\mathrm{P}$ \\
\hline Zoocoria & 0,807 & 0,668 \\
\hline Anemocoria & 4,155 & 0,125 \\
\hline Autocoria & 0,435 & 0,804 \\
\hline \multicolumn{3}{|l|}{ Indivíduos } \\
\hline Zoocoria & 0,911 & 0,634 \\
\hline Anemocoria & 9,306 & $0,009^{x}$ \\
\hline Autocoria & 1,330 & 0,514 \\
\hline
\end{tabular}

${ }^{\ddagger}$ Diferença se deve à menor frequência no interior florestal

As épocas de altas porcentagens refletiram principalmente os picos de frutificação de algumas espécies dentre as mais abundantes na área. Por exemplo, a alta frutificação de março a agosto de 2008 se deveu principalmente à Cecropia hololeuca, Cecropia pachystachya, Euterpe edulis, Miconia cinnamomifolia e Sloanea monosperma.

A curva de intensidade ponderada pela área basal apresentou comportamento semelhante aos demais índices, com diferenças de comportamento em alguns meses específicos. Foi registrado um pico de alta intensidade ponderada em maio de 2008. Esse pico está inserido na época de alta atividade e intensidade de Fournier, mas não há coincidência entre os picos das três curvas. As espécies que contribuíram de forma determinante para este pico de alta intensidade ponderada são as mesmas que determinaram o padrão para intensidade de Fournier, porém com o acréscimo de algumas espécies que se destacam pela área basal elevada. Em maio de 2008, ocorreu frutificação de espécies como Cecropia hololeuca $(\mathrm{ab}=0.17)$, Euterpe edulis $(\mathrm{ab}=0.11)$, Myrcia splendens $(\mathrm{ab}=0.11)$ e Psychotria vellosiana $(\mathrm{ab}=0,11)$.

Os fenogramas das espécies anemocóricas mostram presença de frutos maduros ao longo de todos os meses de estudos (Fig. 1B e E). Esse comportamento foi determinado basicamente pela frutificação de Pseudopiptadenia contorta, que apresentou frutos maduros em 21 meses ao longo do trabalho. Duas épocas de alta atividade de frutificação foram registradas na comunidade, de dezembro de 2006 a junho de 2007 e de maio a agosto de 2008 (Fig. 1B). Alta intensidade de Fournier com duração de alguns meses foi registrada apenas de março a maio de 2007, coincidindo com época de alta atividade de frutificação, embora com menor duração (Fig. 1E). Apuleia leiocarpa e Clethra scabra apresentaram alta intensidade de frutificação nessa época de alta intensidade. Afora isso, houve dois outros picos isolados de intensidade de Fournier (Fig. 1E).

Foram registradas cinco épocas muito breves de alta intensidade ponderada pela área basal, o que resultou em uma dinâmica em pulsos para a anemocoria através desse índice (Fig.1E). Isso refletiu em muito a fenodinâmica de frutificação de Pseudopiptadenia contorta, devido ao seu elevado valor de área basal total $(0,31)$, e explica as diferenças entre as curvas de intensidade de Fournier e intensidade ponderada pela área basal (Fig. 1E).

De janeiro a agosto de 2007, com exceção de fevereiro e junho, a alta intensidade ponderada também acompanhou a frutificação de Pseudopiptadenia contorta, cujo valor de área basal total fez com que o índice de intensidade ponderada se elevasse nesses meses. Já a época de maior intensidade de Fournier, de março a maio de 2007, está relacionada à alta intensidade de frutificação das espécies Apuleia leiocarpa e Clethra scabra, demarcando as diferenças nas curvas fenológicas construídas com os dois índices.

As curvas fenológicas das espécies autocóricas se caracterizam pela alternância entre alguns meses de frutificação expressiva com meses de frutificação baixa ou ausente (dinâmica em pulsos, Fig. 1C e F). O pico de atividade foi em novembro de 2006, com a frutificação de quatro espécies (Fig. 1C). As curvas de intensidade de Fournier e ponderada pela área basal tiveram pouca sobreposição (Fig. 1F). A maior intensidade de Fournier foi de setembro e outubro de 


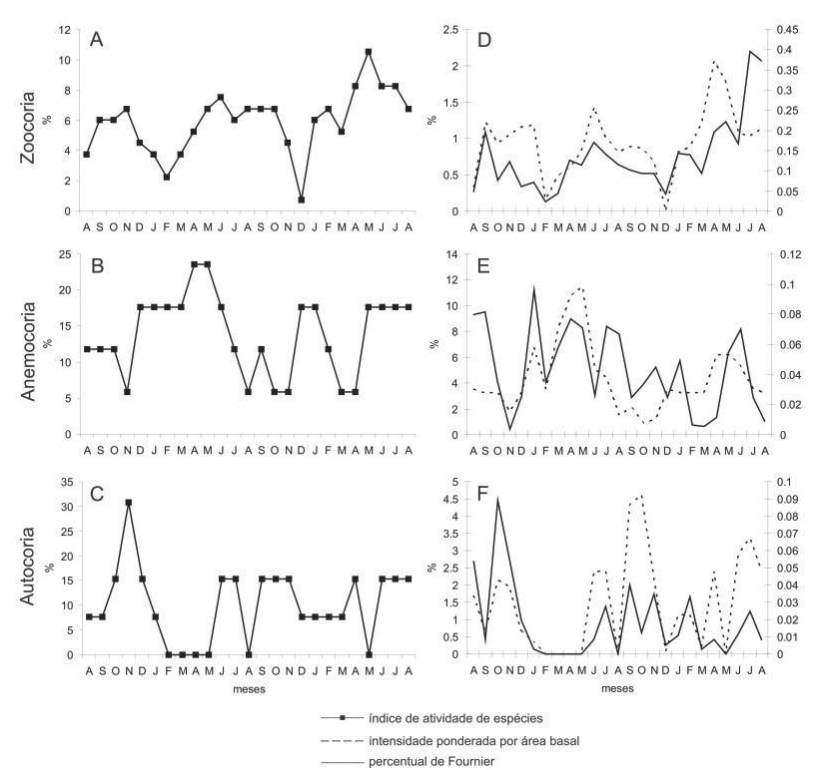

Figuras 1. Fenodinâmica de frutificação de espécies arbóreas com síndrome de dispersão zoocórica (44 espécies), anemocórica (sete espécies) e autocórica (seis espécies) na Reserva Biológica União, sudeste do Brasil, entre agosto de 2006 e agosto de 2008. (A-C) índice de atividade de espécies; (D-F) percentual de intensidade de Fournier (eixo à esquerda) e intensidade de Fournier ponderada pela área basal (eixo à direita).

2007, em que principalmente Bauhinia forficata frutificou com alta intensidade, porém a curva de intensidade ponderada apresentou valores baixos nestes meses, uma vez que esta espécie apresenta valor de área basal total baixo (0.02). Já o pico de intensidade ponderada foi em outubro de 2006, devido à frutificação de duas espécies com valor de área basal total elevado, Mabea fistulifera $(\mathrm{ab}=0.26) \mathrm{e}$ Senefeldera verticillata $(\mathrm{ab}=0.34)$.

Na comparação da fenologia entre os ambientes foi observado que em GA 26 espécies produziram frutos (29,5\% do total), sendo 22 zoocóricas, duas anemocóricas e duas autocóricas. Em RE, 28 espécies frutificaram (33,3\%), sendo 18 zoocóricas, seis anemocóricas e quatro autocóricas e 18 espécies frutificaram no IN (24\%), destas, 13 são zoocóricas, uma anemocórica e quatro autocóricas.

O comportamento das curvas de atividade e intensidade de Fournier para frutificação das espécies zoocóricas diferiu entre os três ambientes, devido aos valores mais altos em RE (Tab. 3, Fig. 2). Nos três ambientes isoladamente, a frutificação não foi contínua, já que houve alguns meses sem frutificação (Fig. 2). Em RE, as espécies zoocóricas apresentaram alta atividade de frutificação em três épocas ao longo do período de estudo: em setembro de 2006, junho de 2007, fevereiro a maio de 2008 (Fig. 2A). Em GA, as épocas de maior atividade foram novembro e dezembro de 2006 e maio a agosto de 2008. A curva de atividade no IN se caracterizou pela alternância entre meses de pouca ou nenhuma frutificação com meses de frutificação alta (pico de atividade em abril de 2008) (Fig. 2A).
O comportamento das curvas de intensidade de Fournier se assemelha ao registrado para atividade fenológica (Fig. 2B). Em RE, além de um evento isolado em setembro de 2006, foram registradas duas épocas de alta intensidade, de março a outubro de 2007 e de fevereiro a julho de 2008 (Fig. 2B). Na primeira época, ocorreu intensa frutificação de Cecropia hololeuca, Cupania racemosa, Couepia schottii, Euterpe edulis, Miconia cinnamomifolia e Psychotria vellosiana. Em GA, houve apenas um pico em dezembro de 2006 (Fig. 2B), com frutificação de Heisteria ovata, Pradosia kuhlmannii, Quiina sp. e Siparuna guianensis. Semelhantemente, o pico em abril de 2008 no IN refletiu a alta intensidade de frutificação de Euterpe edulis, Pouteria bangii e de espécies de Myrtaceae.

A intensidade de frutificação ponderada pela área basal diferiu entre os três ambientes sendo maior em RE e menor em IN (Tab. 3, Fig. 2C). Em RE, houve duas épocas de alta intensidade ponderada, que coincidem com as épocas de alta intensidade de Fournier, porém com menor duração. A alta intensidade entre junho e setembro de 2007 se deveu à intensa frutificação de Astrocaryum aculeatissimum ( $\mathrm{ab}=$ 0,23), Cecropia hololeuca ( $\mathrm{ab}=0,48)$ e Cupania racemosa $(a b=2,25)$. A segunda época de alta frutificação foi de fevereiro a maio de 2008, em que espécies de alto valor de área basal frutificaram intensamente, como Cecropia hololeuca, Cupania racemosa, Euterpe edulis $(\mathrm{ab}=0,25)$ e Psychotria vellosiana $(0,11)$.

As curvas de intensidade ponderada em GA e IN destoam da RE pelos valores muito baixos $(<0,05)$ em vários meses. Em GA foi registrada uma época de maior intensidade de novembro de 2006 a janeiro de 2007, devido à frutificação de espécies com alto valor de área basal, como Heisteria ovata $(\mathrm{ab}=0,29)$, e em maio e junho de 2008, devido à frutificação de Myrcia splendens $(\mathrm{ab}=0,3)$.

Em GA, em novembro e dezembro de 2006, as espécies Pradosia kuhlmannii e Quina sp. tiveram alta intensidade de frutificação, juntamente com Heisteria ovata (representando a época de maior intensidade de Fournier). No entanto, dentre as três espécies, Heisteria ovata é a única que apresenta alto valor de área basal, diferença evidenciada na queda do pico na curva de intensidade ponderada.

\section{Discussão}

A predominância de frutos zoocóricos em detrimento das demais síndromes na comunidade arbórea da Rebio União coincide com o descrito para outras áreas de Mata Atlântica (Morellato \& Leitão-Filho 1992; Tabarelli \& Peres 2002; Kinoshita et al. 2006; Yamamoto et al. 2007) e também com os resultados encontrados na mesma área considerando as plantas de DAP $\geq 5 \mathrm{~cm}$ (Carvalho 2010; Hottz 2010). Mais além, a predominância de zoocoria é um padrão geral para florestas de clima tropical úmido e de baixa sazonalidade climática, enquanto predomínio de anemocoria é observado em florestas de clima seco (Howe \& Smallwood 1982). 
Tabela 3. Valores do teste t pareado não-paramétrico de Wilcoxon para a fenodinâmica de frutificação de espécies arbóreas zoocóricas, medida pelos índices de atividade de espécies, percentual de intensidade de Fournier e intensidade ponderada por área basal, entre os ambientes de bordas de gasoduto (GA) e de rede elétrica (RE) e interior de mata (IN) na Reserva Biológica União, sudeste do Brasil. Valores em negrito representam diferenças significativas para $\mathrm{p}<0,05$.

\begin{tabular}{|c|c|c|c|c|c|c|c|c|c|}
\hline \multirow{2}{*}{\begin{tabular}{l}
\multicolumn{1}{c}{ Parâmetro } \\
Ambientes \\
comparados
\end{tabular}} & \multicolumn{3}{|c|}{ Atividade de espécies } & \multicolumn{3}{|c|}{ Intensidade de Fournier } & \multicolumn{3}{|c|}{$\begin{array}{c}\text { Intensidade ponderada } \\
\text { pela área basal }\end{array}$} \\
\hline & $\begin{array}{l}\text { RE vs. } \\
\text { IN }\end{array}$ & $\begin{array}{l}\text { GA vs. } \\
\text { IN }\end{array}$ & $\begin{array}{l}\text { GA vs. } \\
\text { RE }\end{array}$ & $\begin{array}{l}\text { RE vs. } \\
\text { IN }\end{array}$ & $\begin{array}{l}\text { GA vs. } \\
\text { IN }\end{array}$ & $\begin{array}{l}\text { GA vs. } \\
\text { RE }\end{array}$ & $\begin{array}{l}\text { RE vs. } \\
\text { IN }\end{array}$ & $\begin{array}{l}\text { GA vs. } \\
\text { IN }\end{array}$ & $\begin{array}{c}\text { GA vs. } \\
\text { RE }\end{array}$ \\
\hline$Z$ & 3,82 & 1,57 & 3,97 & 4,11 & 1,57 & 3,48 & 3,91 & 2,05 & 3,45 \\
\hline $\mathrm{P}$ & $<0,001$ & 0,12 & $<0,001$ & $<0,001$ & 0,11 & $<0,001$ & $<0,001$ & 0,03 & $<0,001$ \\
\hline
\end{tabular}

Na Rebio União, os três ambientes não diferiram na distribuição de espécies de diferentes síndromes, com espécies zoocóricas predominando tanto nas bordas quanto no interior florestal. Espécies anemocóricas são constantemente relacionadas a ambientes abertos, secos e com maior influência dos ventos, devido às características de seus frutos, comumente secos e deiscentes com sementes aladas e aerodinâmicas ou com os próprios frutos indeiscentes e aerodinâmicos (Howe \& Smallwood 1982; Jara-Guerrero et al. 2011). Essa associação levou a estudos sobre fragmentação de habitats, cuja hipótese era de que haveria mais espécies anemocóricas em ambientes de borda em comparação ao interior (e.g., Tabarelli et al. 1999; Nunes et al. 2003; Carvalho 2010). No entanto, a ocorrência de espécies com distintas estratégias de dispersão pode depender do estrato analisado (Jara-Guerrero et al. 2011). Por exemplo, Yamamoto et al. (2007) identificou predominância de zoocoria nos estratos médios e inferiores e de anemocoria e autocoria em estratos superiores, devido à maior presença de vento e maiores temperaturas. Esses padrões podem acarretar em semelhanças na distribuição das síndromes entre bordas e interiores quando os estratos são analisados indistintamente, como no presente estudo.

Uma hipótese alternativa é que em bordas há predominância de espécies pioneiras não tolerantes à sombra, possivelmente presentes no banco de sementes antes da abertura da floresta ou recrutadas posteriormente e, por outro lado, árvores emergentes, de grandes sementes e tolerantes à sombra, diminuem em abundância local (Tabarelli \& Peres 2002). Isso acarreta que as diferenças entre bordas e interior não se deem no aumento de anemocoria nas bordas, mas sim de espécies com frutos zoocóricos pequenos, associados à dispersão por aves pequenas, em detrimento de frutos associados a mamíferos ou aves com abertura bucal grande (Cara 2006). Embora nossa análise não tenha diferenciado tipos de frutos zoocóricos, aparentemente não há predomínio de frutos grandes no interior, pois o número de espécies das famílias tipicamente associadas à dispersão por vertebrados grandes (i.e., Lauraceae, Myrtaceae e Sapotaceae) é equivalente entre os ambientes na Rebio União. Assim, a explicação mais provável para a distribuição das síndromes nos distintos ambientes na Rebio União é que os efeitos de bordas lineares formadas por corredores de gasoduto e rede elétrica seriam menos rígidos que os efeitos tipicamente observados em fragmentos completamente circundados por matrizes extensas (e.g., Laurance et al. 2006). Especifi- camente na Rebio União, a largura da matriz é inferior a 30 $\mathrm{m}$ no caso da borda de gasoduto e a matriz nas bordas de rede elétrica é formada por vegetação arbustiva-arbórea, o que pode ter amenizado diferenças entre borda e interior em relação aos fatores determinantes da distribuição de espécies pioneiras e não-pioneiras. Entretanto, detectamos menor frequência de indivíduos anemocóricos no interior florestal, que pode indicar algum recrutamento diferencial das espécies devido ao efeito de borda.

A fenodinâmica das espécies autocóricas e anemocóricas não se concentrou nos meses mais secos na Rebio União, como observado em outras áreas de Mata Atlântica. A associação de anemocoria com dispersão na estação seca ou na transição da estação seca para a chuvosa é relacionada à menor umidade, maior velocidade dos ventos e eficiência na maturação dos frutos (Jara-Guerrero et al. 2011). Além disso, o desenvolvimento das plantas seria favorecido no início da estação chuvosa, com maior probabilidade de incidência solar, umidade adequada ao desenvolvimento das plântulas e desenvolvimento do sistema radicular (Janzen 1967; Morellato \& Leitão-Filho 1992). A amostragem de espécies anemocóricas e autocóricas na Rebio União (respectivamente, sete e seis espécies frutificaram) pode ter sido muito baixa para representar o comportamento fenológico dessas guildas na comunidade. Por exemplo, as únicas espécies anemocóricas que frutificaram na borda de gasoduto e no interior pertencem ao gênero Pseudopiptadenia ( $P$. contorta e $P$. warmingii, Fabaceae), as quais apresentam frutificação de longa duração, determinada pela maturação gradual, que resulta na deiscência vagarosa e contínua de seus frutos (Pires \& Freitas 2007).

A disponibilidade de frutos zoocóricos maduros ao longo de todo o ano na Rebio União, mesmo que em baixa atividade e intensidade, pode estar associada à manutenção de recursos para animais frugívoros. Tal explicação tem sido proposta para outras florestas tropicas ombrófilas ou semidecíduas (Hilty 1980; Morellato et al. 1989; Morellato \& Leitão-Filho 1992; Talora \& Morellato 2000). Já alguns outros estudos observaram maior número de espécies zoocóricas com dispersão na época mais úmida do ano, o que supostamente favoreceria a duração e atratividade dos frutos (Yamamoto et al. 2007; Jara-Guerrero et al. 2011). Em ambos os casos, os fatores causais propostos como explicação para os distintos padrões não foram claramente testados, além de haver possíveis limitações em relação a flutuações temporais e uso da fenodinâmica de espécies como estimativa da dis- 
Índice de atividade de espécies

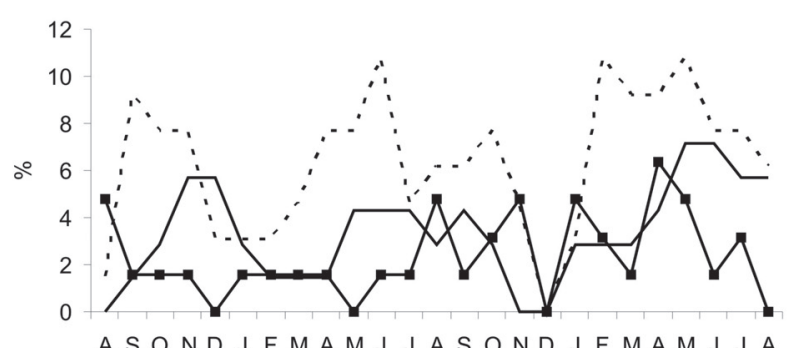

Percentual de intensidade Fournier

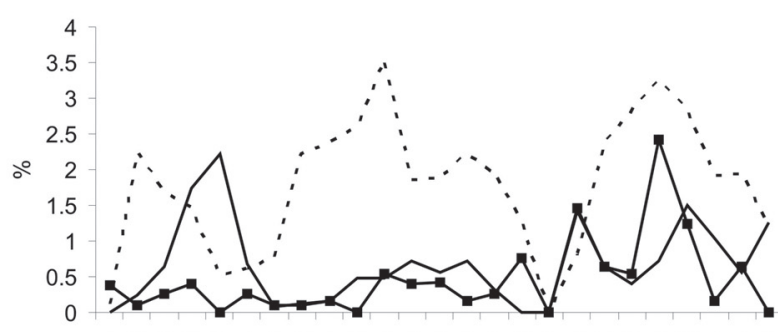

A SONDJFMAMJJASONDJFMAMJJA

Intensidade ponderada por área basal

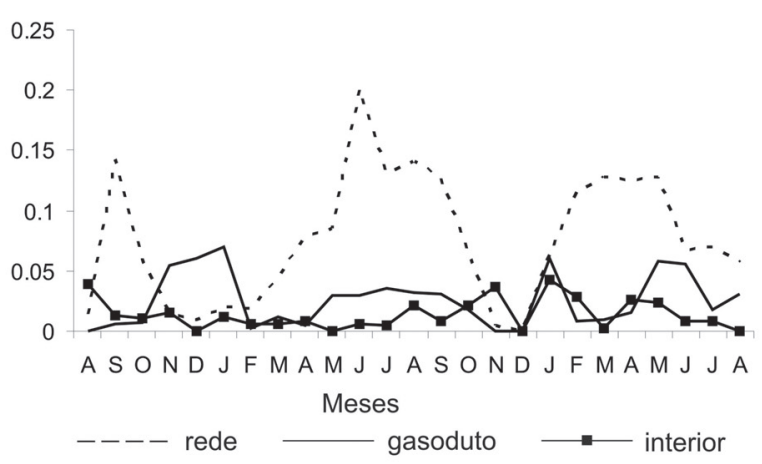

Figura 2. Fenodinâmica de frutificação de 44 espécies arbóreas zoocóricas em ambientes de bordas lineares (gaseoduto e rede elétrica) e interior da mata na Reserva Biológica União, sudeste do Brasil, entre agosto de 2006 e agosto de 2008.

ponibilidade de recursos aos frugívoros, dada as diferenças na abundância das espécies (q.v., Pires 2010).

Os ambientes de borda e interior na Rebio União diferem em estrutura e composição de espécies, o que pode ser indicativo de que os efeitos de borda locais têm alterado estruturalmente a comunidade de árvores (Rodrigues 2004), refletindo ainda nas diferenças encontradas no comportamento fenológico (Pires 2010). A distribuição de árvores de grande porte (altura e área basal) difere entre os ambientes de interior (com maior presença neste, $25 \%$ ) e bordas GA e RE (15\%). No entanto, quando comparamos os ambientes quanto à fenodinâmica com enfoque nos modos de dispersão, não encontramos diferenças claras. As mudanças estruturais na comunidade parecem não ser suficientes para que, neste nível de comparação, diferenças na fenodinâmica sejam significativas. Este fato pode ser compreendido pelas características de bordas lineares, nas quais os efeitos de borda atuariam de forma menos impactante.

As espécies zoocóricas frutificaram com baixa intensidade ao longo do estudo, sendo os maiores índices de intensidade em borda RE. Já foi registrado que espécies arbóreas podem ter sua produção total de frutos afetada pelo efeito da fragmentação tanto com aumento na produção, quando comparada a áreas de mata contínua (Herrerías-Diego et al. 2006), quanto não sofrerem efeito (Fuchs et al. 2003). Por exemplo, a produção total de frutos por Pachira quinata não foi afetada pela fragmentação em floresta tropical seca, no entanto, a espécie produziu o dobro de flores em áreas isoladas que em contínuas, de modo que a relação entre fruto maduro e flor foi negativa (Fuchs et al. 2003). Além disso, Rodrigues (2004) também identificou maior diversidade e riqueza de espécies em borda RE na Rebio União e propôs que este resultado fosse explicado pela hipótese dos distúrbios intermediários (sensu Connell 1978). Tal hipótese sugere que ambientes sujeitos a distúrbios com frequiência intermediária e sem elevada intensidade tenderiam a apresentar maior diversidade. Como colocado por Pires (2010), no ambiente de RE, o corte seletivo de espécies dominantes, de grande porte, manteria um nível intermediário de perturbação, enquanto no ambiente de borda GA, a capina constante levaria a perturbações maiores e de elevada freqüência. Esses resultam reforçam o indicativo de que os efeitos de borda são atenuados em bordas lineares e dependentes do manejo aplicado nessas bordas.

Em conclusão, nossos resultados indicam a existência de efeitos de borda na fenodinâmica de frutificação de espécies zoocóricas, porém ausência desses efeitos na distribuição das síndromes de dispersão. Isso sugere que a passagem de redes elétricas e gasodutos em remanescentes de vegetação extensos e bem preservados pode afetar a dinâmica temporal de disponibilidade de frutos para frugívoros, com possíveis consequências para as interações planta-dispersor, mesmo quando não acarretam em diminuição na oferta total de recursos para esses animais.

\section{Agradecimentos}

Ao CNPq pela concessão de bolsa de iniciação científica (PIBIC) a GR e de produtividade em pesquisa (PQ) a LF. À FAPERJ pela concessão de bolsa de doutorado a JPAP. À Reserva Biológica União pelo suporte no trabalho de campo e ao Instituto Nacional de Metereologia (INMET) por disponibilizar dados climáticos.

\section{Referências bibliográficas}

Aizen, M. \& Feinsinger, P. 1994. Forest fragmentation, pollination, and plant reproduction in Chaco dry forest, Argentina. Ecology 75: 330- 351.

Almeida-Neto, M.; Campassi, F.; Galetti, M.; Jordano, P. \& Oliveira-Filho, A. 2008. Vertebrate dispersal syndromes along the Atlantic forest: broad-scale patterns and macroecological correlates. Global Ecology and Biogeography 17: 503-513. 
Bencke, C.S.C. \& Morellato, L.P.C., 2002. Comparação de dois métodos de avaliação da fenologia de plantas, sua interpretação e representação. Revista Brasileira de Botânica 25: 269-275.

Cara, P.A.A. 2006. Efeito de borda sobre a fenologia, as síndromes de polinização e a dispersão de sementes de uma comunidade arbórea na Floresta Atlântica ao norte do Rio São Francisco. Tese de doutorado. Recife, Universidade Federal de Pernambuco.

Carvalho, F.A. 2010. Síndromes de dispersão de espécies arbóreas de florestas ombrófilas submontanas do estado do Rio de Janeiro. Revista Árvore 34: 1017-1023.

Connell, J.H. 1978. Diversity in tropical rain forest and coral reefs. Science 199: 1302-1310.

Corlett, R.T. \& LaFrankie, J.V. 1998. Potential impacts of climate change on tropical Asian forest through an influence on phenology. Climatic Change 39: 439-453.

CRIA-FAPESP. 2003. NEOFRUG - Banco de Dados de Frugivoria Neotropical. Disponível em: <http://www.cria.org.br/neofrug> (Acesso em 01/07/2011).

Cunningham, S.A. 2000. Effects of habitat fragmentation on the reproductive ecology of four plant species in Mallee woodland. Conservation Biology 14: 758-768.

Doligez, A. \& Joly, H.I. 1997. Genetic diversity and spatial structure within a natural stand of a tropical forest tree species, Carapa procera (Meliaceae), in French Guiana. Heredity 79:72-82.

Du, YJ.; Mia, XC.; Liua, XJ.; Chena, L. \& Ma, KP. 2009. Seed dispersal phenology and dispersal syndromes in a subtropical broad-leaved forest of China. Forest Ecology and Management 258: 1147-1152.

Fournier, L.A. 1974. Un método cuantitativo para la medición de características fenológicas en árboles. Turrialba 24: 422-423

Fuchs, E.J.; Lobo, J.A. \& Quesada, M. 2003. Effects of forest fragmentation and flowering phenology on the reproductive success and mating patterns of the tropical dry forest tree Pachira quinata. Conservation Biology 17:149-157.

Galetti, M. \& Pizo, M.A. 1996. Fruit eating by birds in a forest fragment in southeastern Brazil. Ararajuba 4: 71-79

Goosem, M. 1997. Internal fragmentation: the effects of roads, highways and powerline clearings on movements and mortality of rainforest vertebrates. Pp.241-255. In: Laurance, W.F. \& Bierregaard, R.O. (Eds.). Tropical forest remnants: ecology, management, and conservation of fragmented communities. Chicago, University of Chicago Press.

Herrerías-Diego, Y.; Quesada, M.; Stoner, K.E. \& Lobo, J.A. 2006. Effects of forest fragmentation on phenological patterns and reproductive success of the tropical dry forest tree Ceiba aesculifolia. Conservation Biology 20: 1111-1120.

Hilty, S.L. 1980. Flowering and fruiting periodicity in a premontane rain forest in Pacific Colombia. Biotropica 12: 292-306.

Hottz, D. 2010. Efeitos de borda sobre comunidades arbóreas em dois remanescentes florestais de Mata Atlântica: composição florística e síndromes de dispersão de sementes. Dissertação de mestrado. Rio de Janeiro, Museu Nacional, Universidade Federal do Rio de Janeiro.

Howe, H.F. \& Smallwood, J. 1982. Ecology of seed dispersal. Annual Review of Ecology and Systematics 13: 201-228.

Janzen, D.H. 1967. Syncronization of sexual reproduction of trees within the dry season in Central America. Evolution 21: 621-637.

Jara-Guerrero, A.; De la Cruz, M; \& Méndez, M. 2011. Seed dispersal spectrum of woody species in south Ecuadorian dry forests: environmental correlates and the effect of considering species abundance. Biotropica 43: 722-730.

Jordano, P.; Galetti, M.; Pizo, M.A.; Silva, W.R. 2006. Ligando frugivoria e dispersão de sementes à biologia da conservação. Pp. 411-436. In: Duarte, C.F.; Bergallo, H.G.; Van Sluys, M.\& Alves, M.A.S. (Orgs.). Biologia da conservação: essências. São Carlos, Editora Rima.

Kapos, V.; Wandelli, E.; Camargo, J.L. \& Ganade, G. 1997. Edge-related changes in environment and plant responses due to forest fragmentation in Central Amazonia. Pp. 33-44. In: Laurance, W.F. \& Bierregaard, R.O. (Eds.). Tropical forest remnants: ecology, management and conservation of fragmented communities. Chicago, University of Chicago Press.

Kinoshita, L.S.; Torres, R.B.; Forni-Martins, E.R.; Spinelli, T.; Ahn, Y.J. \& Constâncio, S.S. 2006. Composição florística e síndromes de polinização e de dispersão da mata do Sítio São Francisco, Campinas, SP. Acta Botanica Brasilica 20: 313-327.

Laurance, W.F.; Rankin-De-Merona, J.M.; Andrade, A.; Laurance, S.G.; D’Angelo S.; Lovejoy T.E. \& Vasconcelos, H.L. 2003. Rain-forest fragmentation and the phenology of Amazonian tree communities. Journal of Tropical Ecology 19: 343-347.

Laurance W.F.; Nascimento H.E.M.; Laurance S.G.; Andrade A.; Fearnside P.M.; Ribeiro J.E.L.; Chave J. \& Muller-Landau, H. 2006. Rain forest fragmentation and the proliferation of successional trees. Ecology 87: $469-482$

Morellato, L.P.C.\& Leitão-Filho, H. F. 1992. Padrões de frutificação e dispersão na serra do Japi. Pp.112-140. In: Morellato, L.P.C. (Ed.). História Natural da Serra do Japi: ecologia e preservação de uma área florestal no Sudeste do Brasil. Campinas, Editora da Unicamp.

Morellato L.P.C; Rodrigues, R.R.; Leitão-Filho, H.F. \& Joly, C.A. 1989. Estudo comparativo da fenologia de espécies arbóreas de floresta de altitude e floresta mesófila semidecídua na Serra do Japi, Jundiaí, São Paulo. Revista Brasileira de Botânica 12: 85-98.

Morellato, L.P.C.; Talora, D.C.; Takahasi, A.; Bencke, C.C.; Romera, E.C. \& Zipparro, V.B. 2000. Phenology of Atlantic Forest trees: a comparative study. Biotropica 32: 811-823.

Murawski, D.A.; Hamrick, J.L.; Hubbell, S.P. \& Foster, R.B. 1990. Mating system of two Bombacaceous trees of a neotropical moist forest. Oecologia 82: 501-506.

Murcia, C. 1995. Edge effects in fragmented forests: implications for conservation. Trends in Ecology and Evolution 10: 58-62.

Nunes, Y.R.F.; Mendonça, A.V.R.; Oliveira-Filho, A.T.; Botezelli, L. \& Machado, E.L.M. 2003. Variações da fisionomia, diversidade e composição de guildas da comunidade arbórea em um fragmento de floresta semidecidual em Lavras, MG. Acta Botanica Brasilica 17: 213-229.

Oliveira-Filho, A.T.; Carvalho, D.A.; Vilela, E.A.; Curi, N. \& Fontes, M.A.L. 2004. Diversity and structure of the tree community of a fragment of tropical secondary forest of the Brazilian Atlantic Forest domain 15 and 40 years after logging. Revista Brasileira de Botânica 27: 685-701.

Pires, J.P.A. 2010. Fenologia do componente arbóreo em bordas lineares e interior em um remanescente de Floresta Atlântica. Tese de doutorado. Jardim Botânico do Rio de Janeiro, Rio de Janeiro

Pires, J.P.A. \& Freitas, L. 2007. Fenodinâmica reprodutiva de Pseudopiptadenia (Leguminosae- Mimosoideae) em uma área de Mata Atlântica Montana. Natureza on Line 5: 48-54.

Pohlman, C.L.; Turton, S.M. \& Goosem, M. 2007. Edge effects of linear canopy openings on tropical rain forest understory microclimate. Biotropica 39: 62-71.

Ramos, F.N. \& Santos, F.M. 2005. Phenology of Psychotria tenuinervis (Rubiaceae) in Atlantic Forest fragment. Canadian Journal of Botany 83: 1305-1316.

Restrepo, C.; Gomez, N. \& Heredia S. 1999. Anthropogenic edges, treefall gaps, and fruit-frugivore interactions in a neotropical montane forest. Ecology 80: 668-685.

Reys, P.; Galetti, M.; Morellato, L.P.C. \& Sabino, J. 2005. Fenologia reprodutiva e disponibilidade de frutos de espécies arbóreas em mata ciliar no Rio Formoso, Mato Grosso do Sul. Biota neotropica 5: 309-318.

Rodrigues, P.J.F.P. 2004. A vegetação da Reserva Biológica União e os efeitos de borda na Mata Atlântica fragmentada. Tese de doutorado. Universidade Estadual do Norte Fluminense, Campos dos Goytacazes

Santos, H.G.; Anjos, L.H.C.; Oliveira, V.A.; Oliveira, J.B.; Coelho, M.R.; Lumbrelas, J.F. \& Cunha, T.J.F. 2006. Sistema brasileiro de classificação de solos. 2 ed. Rio de Janeiro, EMBRAPA.

Silva, J. M.C. \& Tabarelli, M. 2000. Tree species impoverishment and the future flora of the Atlantic forest of northeast Brazil. Nature 404: 72-73.

Slik, J.W.F.; van Beek, M.; Bernard, C.; Bongers, F.; Breman, F.C.; Cannon, C.H. \& Sidiyasa, K. 2011. Limited edge effects along a burnedunburned Bornean forest boundary seven years after disturbance. Biotropica 43: 288-298.

Spina, A.P.; Ferreira, W.M. \& Leitão-Filho, H.F. 2001. Floração, frutificação e síndromes de dispersão de uma comunidade de floresta de brejo na região de Campinas (SP). Acta Botanica Brasilica 15: 349-368. 
Tabarelli, M. \& Peres, C.A. 2002. Abiotic and vertebrate seed dispersal in the Brazilian Atlantic forest: implications for forest regeneration. Biological Conservation 106: 165-176.

Tabarelli, M.; Mantovani, W. \& Peres, C.A. 1999. Effects of habitat fragmentation on plant guild structure in the montane Atlantic Forest of southeastern Brazil. Biological Conservation 91: 119-127.

Talora, D.C. \& Morellato, P.C. 2000. Fenologia de espécies arbóreas em floresta de planície litorânea do sudeste do Brasil. Revista Brasileira de Botânica 23: 13-26.

van der Pijl, L. 1982. Principles of dispersal in higher plants, 3 ed. Berlim, Springer-Verlag. van Schaik, C.P.; Terborgh, J.W. \& Wright, S.J. 1993. The phenology of tropical forests: adaptive significance and consequences for primary consumers. Annual Review of Ecology and Systematics 24: 353-377.

Veloso, H.P.; Rangel Filho, A.L.R \& Lima, J.C.A. 1991. Classificação da vegetação brasileira, adaptada a um sistema universal. Rio de Janeiro, Instituto Brasileiro de Geografia e Estatística.

Yamamoto, L.F.; Kinoshita, L.S. \& Martins, F.R. 2007. Síndromes de polinização e de dispersão em fragmentos da Floresta Estacional Semidecídua Montana, SP, Brasil. Acta Botanica Brasilica 21: 553-573. 